\title{
Mean arterial pressure nonlinearity in an elastic circulatory system subjected to different hematocrits
}

\author{
Tom Branigan • Diogo Bolster • \\ Beatriz Y. Salazar Vázquez • Marcos Intaglietta • \\ Daniel M. Tartakovsky
}

Received: 24 April 2010 / Accepted: 14 September 2010 / Published online: 29 September 2010

(C) The Author(s) 2010. This article is published with open access at Springerlink.com

\begin{abstract}
The level of hematocrit (Hct) is known to affect mean arterial pressure (MAP) by influencing blood viscosity. In the healthy population, an increase in Hct (and corresponding increase in viscosity) tends to raise MAP. However, data from a clinical study of type 2 diabetic patients indicate that this relationship is not universal. Instead, individuals in the lower levels of Hct range display a decrease in MAP for a given rise in Hct. After reaching a minimum, this trend is reversed, so that further increases in Hct lead to increases in MAP. We hypothesize that this anomalous behavior occurs due to changes in the circulatory autoregulation mechanism. To substantiate this hypothesis, we develop a physically based mathematical model that incorporates autoregulation mechanisms. Our model replicates the anomalous U-shaped relationship between MAP and Hct found in diabetic patients in the same range of Hct variability.
\end{abstract}

T. Branigan

School of Medicine, Royal College of Surgeons, Dublin, Ireland

D. Bolster

Department of Civil Engineering and Geological Sciences,

University of Notre Dame, Notre Dame, Indiana, USA

B. Y. S. Vázquez

Faculty of Medicine, Universidad Juárez del Estado de Durango,

Victoria de Durango, Dgo, Mexico

B. Y. S. Vázquez · M. Intaglietta ( $\varangle)$

Department of Bioengineering, University of California, 9500 Gilman Dr, San Diego, La Jolla, CA 92093-0412, USA

e-mail: mintagli@ucsd.edu

D. M. Tartakovsky

Department of Mechanical and Aerospace Engineering,

University of California, San Diego, La Jolla, CA, USA
Keywords Blood pressure - Hematocrit - Blood viscosity · Model · Diabetes · Blood vessels · Elastic properties · Hypertension · Hypotension · Autoregulation

$\begin{array}{ll}\text { Nomenclature } \\ P & \text { Pressure } \\ P_{\mathrm{Z}} & \text { Pressure gradient } \\ T & \text { Tension } \\ r & \text { Vessel radius } \\ A & \text { Activation } \\ C_{j} & \text { Constant associated with the subscript } j \\ r_{0} & \text { Reference vessel radius } \\ S_{\mathrm{t}} & \text { Stimulus } \\ \sigma & \text { Shear stress } \\ Q & \text { Flow rate } \\ \mu & \text { Effective blood viscosity } \\ \text { MAP } & \text { Mean arterial pressure } \\ \text { Hct } & \text { Hematocrit }\end{array}$

\section{Introduction}

Mean arterial pressure (MAP) depends on physical factors including blood volume, elastic properties of the circulatory tree, length and radius of blood vessels, and blood viscosity, a direct function of hematocrit (Hct). In this work, we focus exclusively on the influence of hematocrit. According to Poiseuille's law, vascular resistance is predominantly determined by vessel diameter, which in healthy individuals is controlled by vascular smooth muscle tone. The latter, in turn, depends on myogenic and chemical autoregulation, influenced by extrinsic neurological and hormonal controls (Levick 2003). Under physiological conditions, the variability of blood viscosity is not sufficient to produce anomalous changes in MAP (Salazar-Vázquez et al. 2006). 
However, Martini et al. (2005) found that MAP decreased significantly when Hct was raised acutely in healthy hamsters, leading to a U-shaped MAP versus Hct distribution-a finding attributed to the competition between nitric oxide (NO) production by shear stress due to changes in blood viscosity and changes in resistance due to changes in blood viscosity. Salazar-Vázquez et al. (2006) found a similar relationship in type 2 diabetic patients, when MAP was plotted as a function of the naturally occurring variability of Hct in the sampled population. Both studies show that there is a range of Hct where increases of Hct cause MAP to decrease, suggesting the presence of blood-rheology sensing mechanisms.

Autoregulation of vascular tone is either attenuated or absent under conditions of endothelial dysfunction, e.g., in the case of endothelial and intimal damage characteristic of diabetes. Diabetic angiopathy lowers myogenic autoregulation through increased collagen cross-linkage by increasing vascular stiffness (Cameron and Cruickshank 2007). The latter results in secondary hypertension and a MAP that is raised sufficiently to maximize the myogenic response (Carlson et al. 2008). The shear stress response is impeded by the deposition of advanced glycation end products on intact extracellular matrix which can absorb, hinder diffusion of, and inactivate NO (Shore and Tooke 1997). Glycated hemoglobin, free radicals, and oxidized low-density lipoproteins also quench the effect of NO. In diabetics, autonomic autoregulation is impaired as shown by the deficient endotheliumdependent relaxation to acetylcholine and bradykinin (Malik et al. 2005).

Under these conditions, microcirculatory regulation resembles that of a system of rigid tubes. Blood viscosity affects peripheral resistance and hence MAP. Maintaining flow rates requires varying driving pressure in proportion to changes in viscosity. The behavior of pressure in the circulation also approximates that within a system of rigid tubes for extreme changes of blood viscosity, such as pathologically high Hcts (Bertinieri et al. 1998; Jefferson et al. 2002) and extreme Hct reductions (Toy et al. 2000).

In an elastic tube, MAP and blood viscosity are related in part by the material properties of the tube (vessel) wall, the viscosity of the flowing fluid, and the tube's autoregulatory capacity. The relationship between changes in Hct and average pressure in an elastic tube is explored by analyzing the interactions between vessel wall mechanics and the pressure distribution in blood vessels at different viscosities.

We use mathematical modeling to investigate the relationship between pressure and the viscosity of a fluid flowing in an autoregulating elastic tube. The model employs parameters and boundary conditions related to the arteriolar circulation and sheds new light on the anomalous U-shaped MAP-Hct distribution found in diabetic patients.

\section{Mathematical model}

Standard analytical models of blood flow in vessels account for their elasticity by invoking the concept of compliance (Loscalzo and Schafer 2002; Secomb 2008). Such models typically result in monotonically increasing relations between pressure and Hct. The proposed autoregulatory elastic compliance model illustrates that under certain conditions, the MAP-Hct curve can exhibit a U-shape observed experimentally by Salazar-Vázquez et al. (2006).

Our model accounts for the possibility of such a behavior by invoking two postulates. First, the degree to which a blood vessel dilates or constricts in response to changes in blood pressure is determined, at least in part, by its elastic properties. Second, pressure at the tube inlet varies with the vessel inner radius in an autoregulatory fashion. The proposed autoregulatory elastic compliance model allows for an arbitrary dependence of blood viscosity on Hct and is valid for either linear or pseudo-linear descriptions of the blood vessel, with both descriptions yielding analogous MAP versus Hct dependence.

We develop a mathematical model that describes how blood pressure $P$ changes with hematocrit (Hct), in a manner that accounts for the elasticity of blood vessels and incorporates various autoregulation mechanisms. We assume steadystate blood flow with constant volumetric flow rate $Q$ in a single blood vessel. This approximation is justified by the very definition of autoregulation which is "the maintenance of a constant supply of blood to an organ in spite of varying arterial pressure"1. Furthermore, we focus on blood vessels whose effective inner radii are sufficiently large for the Stokes equation to provide an adequate description of blood flow. This is a standard approach that can be found in, e.g., Chapter 17 in Loscalzo and Schafer (2002) and Section 5.1 in Keener and Sneyd (2004).

Under these assumptions, the volumetric flow rate is estimated by Poiseuille's law,

$$
Q=-\frac{\pi r^{4} P_{\mathrm{z}}}{8 \mu},
$$

where $r$ is the radius of a blood vessel, $P_{\mathrm{z}}$ is the axial pressure gradient along the vessel, and $\mu$ is the effective blood viscosity. The radius of an elastic blood vessel, $r$, is determined by a balance between blood pressure $P$ and tension in the vessel wall, $T$ from Laplace's law for thin-walled vessels (Carlson et al. 2008; Carlson and Secomb 2005; Secomb 2008),

$P=\frac{T}{r}$.

The tension in the vessel wall, $T$, consists of passive ( $\left.T_{\text {passive }}\right)$ and active $\left(T_{\text {active }}\right)$ components and can be represented as

\footnotetext{
${ }^{1}$ http://www.merriam-webster.com/medlineplus/autoregulation.
} 
their weighted sum,

$T=T_{\text {passive }}+A T_{\text {active }}$,

where $A$ is the fraction of the total autoregulatory potential being exerted by the vessel (i.e., a number between 0 and 1). The passive tension is a function of the vessel's mechanical properties, and the active tension reflects the vessel's autoregulatory capacity.

Following Carlson et al. (2008), Carlson and Secomb (2005), Secomb (2008), we adopt the following phenomenological relations for $T_{\text {passive }}, T_{\text {active }}$, and $A$. The passive component of tension $T_{\text {passive satisfies an exponential model, }}$

$T_{\text {passive }}=C_{\text {passive }} \exp \left[C_{\text {passive }}^{\prime}\left(r / r_{0}-1\right)\right]$,

which, similar to the often-used linear compliance model, reflects a monotonic relation between passive tension and radius, i.e. any increase in tension causes an increase in radius. The active component of tension $T_{\text {active }}$ is described with a Gaussian model,

$T_{\text {active }}=C_{\text {active }} \exp \left[-\left(\frac{r / r_{0}-C_{\text {active }}^{\prime}}{C_{\text {active }}^{\prime \prime}}\right)^{2}\right]$.

The activation $A$ is related to the intensity of the autoregulatory stimulus $S_{\mathrm{t}}$ via a sigmoidal function

$A=\frac{1}{1+\exp \left(-S_{\mathrm{t}}\right)}$.

According to Levick (2003), the intensity of the autoregulatory stimulus $S_{\mathrm{t}}$ depends primarily on the following three phenomena (although additional ones can easily be included). Myogenic response, $S_{\mathrm{myog}}=C_{\mathrm{myog}} T$, accounts for a vessel's response to changes in intraluminal pressure $P$ (or in accordance with Eq. 2 to changes in tension $T$ ), whereby increased pressure causes smooth muscle stretching. Myogenic autoregulation depends on stretch activated ion channels in vascular smooth muscle that, upon stretching, allow calcium ions to enter, inducing contraction. Shear-dependent response, $S_{\text {shear }}=-C_{\text {shear }} \tau$, represents changes in endothelial NO release (a potent vasodilator) caused by changes in endothelial shear stress $\tau$. Metabolic response $S_{\text {meta }}$ accounts for the fact that tissue hypoxia stimulates formation of vasodilator metabolites, such as NO. This metabolic response of the vasculature to alterations in oxygen demand is proportional to the conducted response signal $S_{\mathrm{CR}}$, so that $S_{\text {meta }}=-C_{\text {meta }} S_{\mathrm{CR}}$. Combining these three response mechanisms, $S_{\mathrm{t}}=S_{\text {myog }}+S_{\text {shear }}+S_{\text {meta }}+C_{\text {tone }}$, where $C_{\text {tone }}$ is a dimensionless constant, Carlson et al. (2008) obtained a phenomenological model for the intensity of the autoregulatory stimulus,

$S_{\mathrm{t}}=C_{\text {myog }} T-C_{\text {shear }} \tau-C_{\text {meta }} S_{\mathrm{CR}}+C_{\text {tone }}$.
The constants $C_{\text {passive }}, C_{\text {passive }}^{\prime}, C_{\text {active }}, C_{\text {active }}^{\prime}, C_{\text {active }}^{\prime \prime}$, $C_{\text {myog }}, C_{\text {shear }}, C_{\text {meta }}$, and $C_{\text {tone }}$ in Eqs. $(4,5,6,7)$ are fitting parameters whose values depend on a vessel's radius. Below we will conduct a sensitivity analysis to quantify the effects of uncertainty in these values on modeling predictions.

Combining Eqs. $(2,3,4,5,6,7)$ yields a relationship between intraluminal blood pressure $P$ and vessel radius $r$,

$$
\begin{aligned}
P= & \frac{C_{\text {passive }}}{r} \exp \left[C_{\text {passive }}^{\prime}\left(r / r_{0}-1\right)\right] \\
+ & \frac{C_{\text {active }}}{r} \frac{1}{1+\exp \left(-C_{\text {myog }} T+C_{\text {shear }} \tau+C_{\text {meta }} S_{\mathrm{CR}}-C_{\text {tone }}\right)} \\
& \times \exp \left[-\left(\frac{r / r_{0}-C_{\text {active }}^{\prime}}{C_{\text {active }}^{\prime \prime}}\right)^{2}\right]
\end{aligned}
$$

which accounts for both the natural elastic properties of blood vessels and autoregulation. To relate intraluminal blood pressure $P$ (and MAP) to blood viscosity $\mu$ (and hematocrit (Hct)), we supplement the static relation (8) with the dynamic equation (1). This step requires both a closure assumption to relate pressure gradient $P_{\mathrm{z}}$ to pressure $P$ and a constitutive law, $\mu=\mu(\mathrm{Hct})$, to relate blood viscosity to hematocrit.

We explore two alternative closures. The first is based on the assumption that any increase in overall pressure is proportional to the increase in pressure drop across a given segment according to

$P \propto P_{\mathrm{Z}}$.

Physically, this approximation implies that a greater resistance to flow requires a greater pressure to maintain flow rate. Combining (1) and (9) yields

$P=C_{\mathrm{p}} \frac{8 Q \mu}{\pi r^{4}}$,

where $C_{\mathrm{p}}$, the constant of proportionality between $P_{\mathrm{z}}$ and $P$, must be fit to data by regression or any other appropriate method. The second closure, which is based on the pressureshear hypothesis of Pries and Secomb (2000), is discussed in Appendix A.

Finally, it remains to specify a functional relationship $\mu=$ $\mu(\mathrm{Hct})$. While the analysis and conclusions presented below are valid for any monotonically increasing functional dependence, in lieu of example we use the power-law relationship (Pries et al. 1992):

$\frac{\mu}{\mu_{\text {plasma }}}=1+B\left[(1-\mathrm{Hct})^{C}-1\right]$,

where $\mu_{\text {plasma }}$ is the viscosity of plasma and values of the experimentally determined constants $B$ and $C$ are given in Table 2 of Pries et al. (1992). Their data for relation (11) spans the range of Hct presented in this study, which is characteristic of the variability of Hct in the healthy population. Applicability of relation (11) is indicated to extend to patients with polycythemia, whose Hct can exceed 50\%. 


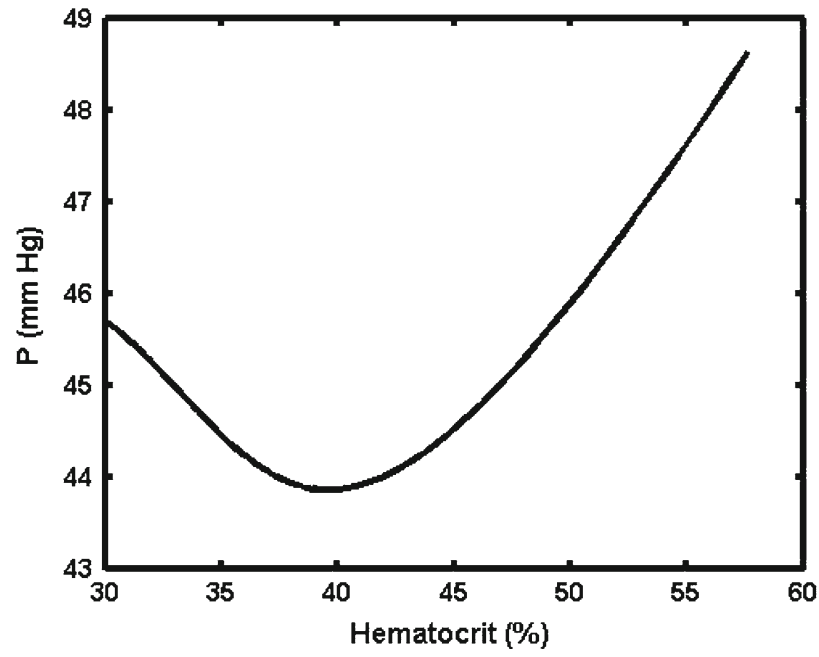

Fig. 1 Relationship between pressure (MAP) and Hct in a model of an elastic arteriolar microvessel, whose behavior is dictated by blood viscosity, a function of Hct. Transmural pressure is with reference to the outer pressure in the tissue, which is assumed to be atmospheric

The MAP versus Hct relationship $P(\mathrm{Hct})$ is found by combining Eqs. (8), (10), and (11). The resulting autoregulatory elastic compliance model provides a nonlinear implicit relationship between blood pressure $P$ and vessel radius $r$. We used Matlab's nonlinear solver fsolve to numerically invert this implicit relation. As demonstrated in Fig. 1, this relation exhibits the "anomalous" U-shaped behavior observed by Salazar-Vázquez et al. (2006) in type 2 diabetic patients.

To understand why such behavior is observed in some patient groups but not others, we note that for it to occur, the function $P(\mathrm{Hct})$ must reach its minimum within the physiological range of Hct. The function $P(\mathrm{Hct})$ achieves its minimum at a point for which

$\frac{\mathrm{d} P}{\mathrm{dHct}}=\frac{\mathrm{d} P}{\mathrm{~d} r} \frac{\mathrm{d} r}{\mathrm{~d} \mu} \frac{\mathrm{d} \mu}{\mathrm{dHct}}=0$.

Since both $r=r(\mu)$ in Eq. (10) and $\mu=\mu$ (Hct) in Eq. (11) are monotonically increasing functions, it follows from Eq. (12) that for the relation $P(\mathrm{Hct})$ to exhibit a U-shaped behavior (i.e., to have a minimum within the physiological range of Hct) requires that

$\frac{\mathrm{d} P}{\mathrm{~d} r}=0$.

Nonlinear expression (8) suggests that this condition is, in general, satisfied. Whether $P(\mathrm{Hct})$ exhibits the U-shaped behavior within the physiological range of Hct is determined by the various modeling constants, whose values depend on the blood vessels' elastic properties and autoregulatory capacity. Consequently, we hypothesize that populations that

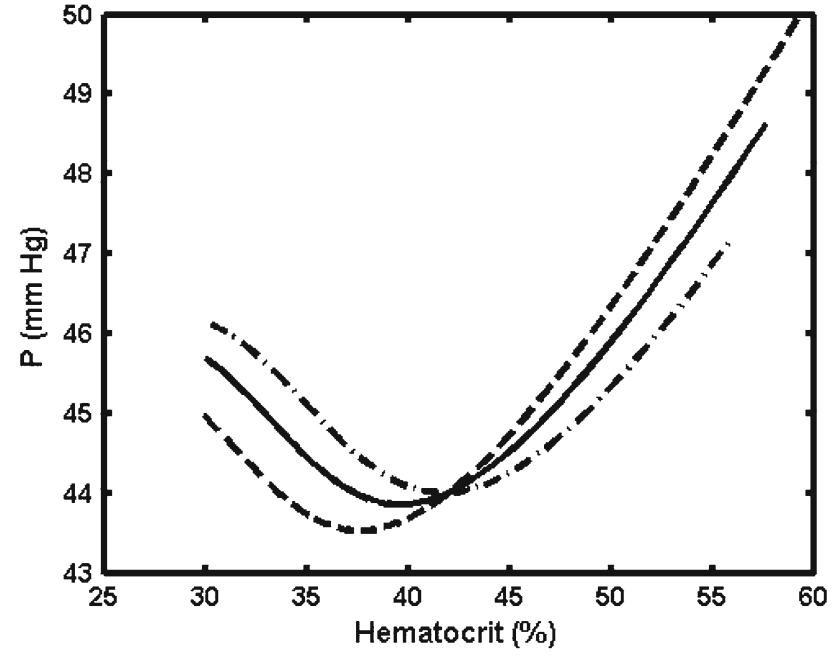

Fig. 2 Sensitivity to $C_{\text {passive }}$, the parameter that characterizes the tension at base conditions, i.e., the tension when $r=r_{0}$. The solid line, corresponding to $C_{\text {passive }}=280 \mathrm{dyn} / \mathrm{cm}$, is the base state in Fig. 1 . The dash-dot and dashed lines correspond to $C_{\text {passive }}=240 \mathrm{dyn} / \mathrm{cm}$ and $C_{\text {passive }}=320 \mathrm{dyn} / \mathrm{cm}$, respectively

exhibit the anomalous MAP-Hct relationship tend to have blood vessels with abnormal elastic and autoregulatory properties. This is likely to be the case for diabetic, aged, etc., populations.

\section{Results}

We used our model to evaluate the equilibrium intraluminal average blood pressure in an elastic autoregulated arteriole-like blood vessel. The reliance on a single blood vessel is based on the assumption that the systemic result is the aggregate of effects at the different levels of branching in the arteriolar microcirculation. While our model allows for both pseudo-elastic descriptions of a blood vessel's wall and an arbitrary functional dependence between blood viscosity and Hct, the results presented in Figs. 1, 2, 3, 4, 5 and 6 correspond to an exponential elastic model (4) and a power-law viscosity-Hct relation (11). The use of nonlinear pseudo-elastic relationships and alternative viscosityHct constitutive models does not alter the predicted behavior. The MAP-Hct relationship presented in Fig. 1 corresponds to $r=50 \mu \mathrm{m}$ and $Q=10 \mathrm{nl} / \mathrm{s}$, both of which are representative of the arterioles of awake hamsters; the rest of the parameters are given in Table 1. These values are deemed representative and similar to those in Carlson et al. (2008). The sensitivity of the proposed model to each of the main fitting parameters is illustrated in Figs. 2, 3, 4 and 5. It is important to realize that, according to expression (8), the results reported in these figures depend largely on the vessel stretching-ratio, $r / r_{0}$, rather than on the vessel radius, $r$, alone. 


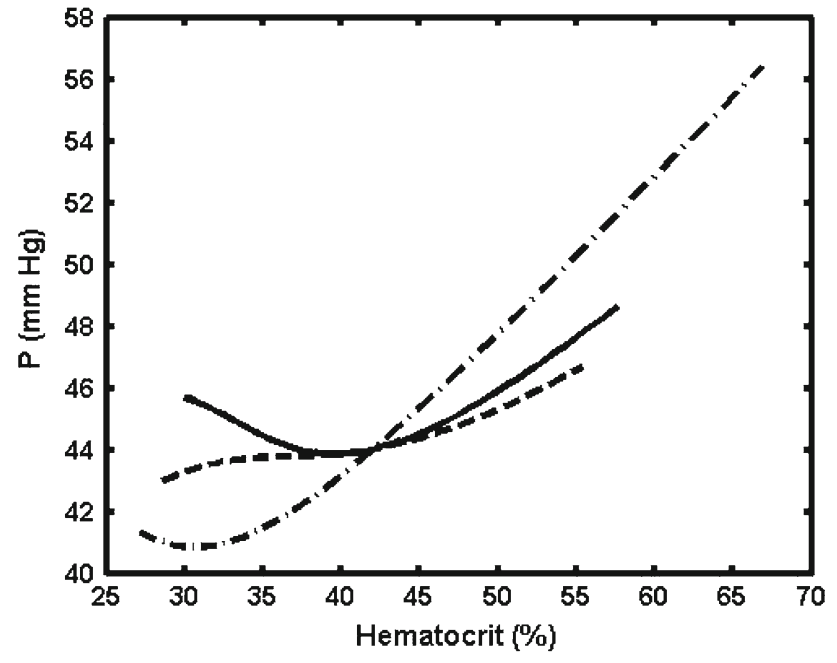

Fig. 3 Sensitivity to $C_{\text {passive }}^{\prime}$, the parameter that accounts for the passive elasticity of the vessel, i.e. the resistance to deformation as the tension changes. The solid line, corresponding to $C_{\text {passive }}^{\prime}=11.5$, is the base state in Fig. 1. The dash-dot and dashed lines correspond to $C_{\text {passive }}^{\prime}=10.5$ and $C_{\text {passive }}^{\prime}=12.5$, respectively

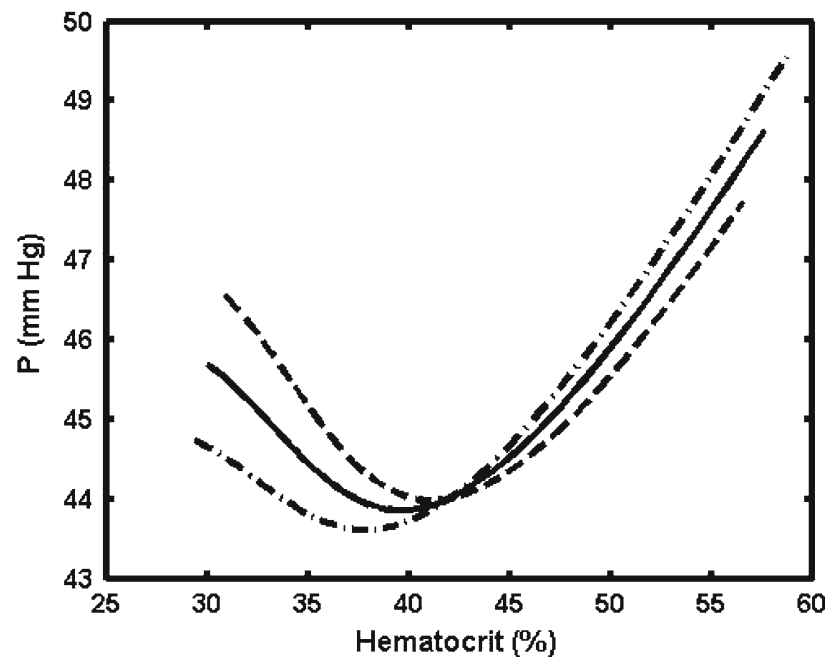

Fig. 4 Sensitivity to $C_{\text {active }}$, the parameter that characterizes the maximum possible value of the active component of tension. The solid line, corresponding to $C_{\text {active }}=275 \mathrm{dyn} / \mathrm{cm}$, is the base state in Fig. 1 . The dash-dot and dashed lines correspond to $C_{\text {active }}=300 \mathrm{dyn} / \mathrm{cm}$ and $C_{\text {active }}=250 \mathrm{dyn} / \mathrm{cm}$, respectively

\section{Discussion}

The principal finding of our study is that the anomalous U-shaped dependence of MAP on Hct found in type 2 diabetic patients (Natali et al. 2005; Salazar-Vázquez et al. 2006) can be modeled by varying Hct (and thus, viscosity) of blood flowing in an elastic tube.

There is substantial literature on the analysis of flow relationships in elastic tubes, most of which is numerical (Quarteroni et al. 2000). Standard analytical models of blood

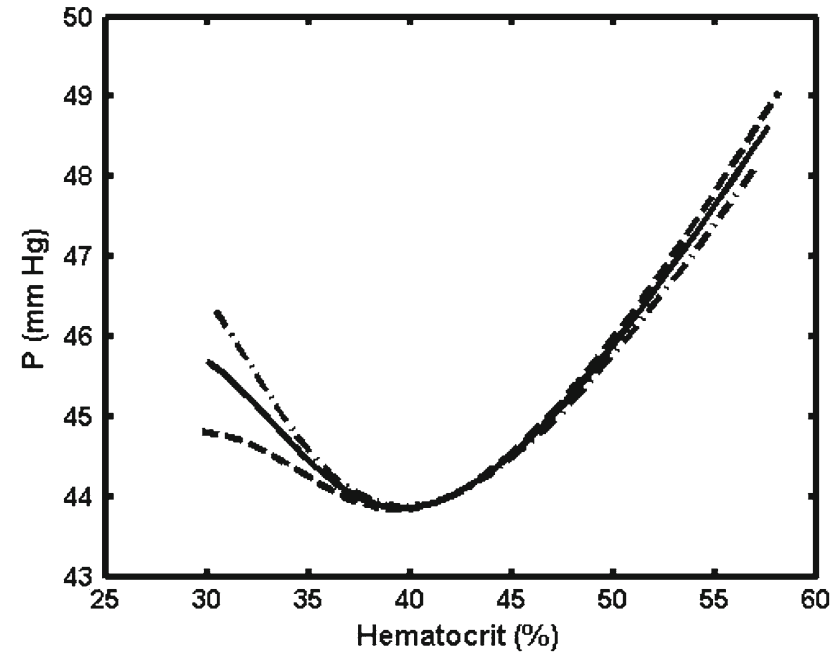

Fig. 5 Sensitivity to $C_{\text {active }}^{\prime}$, the parameter that determines the radius at which the maximum active tension occurs. The solid line, corresponding to $C_{\text {active }}^{\prime}=0.75$, is the base state in Fig. 1. The dash-dot and dashed lines correspond to $C_{\text {active }}^{\prime}=0.45$ and $C_{\text {active }}^{\prime}=1.05$, respectively

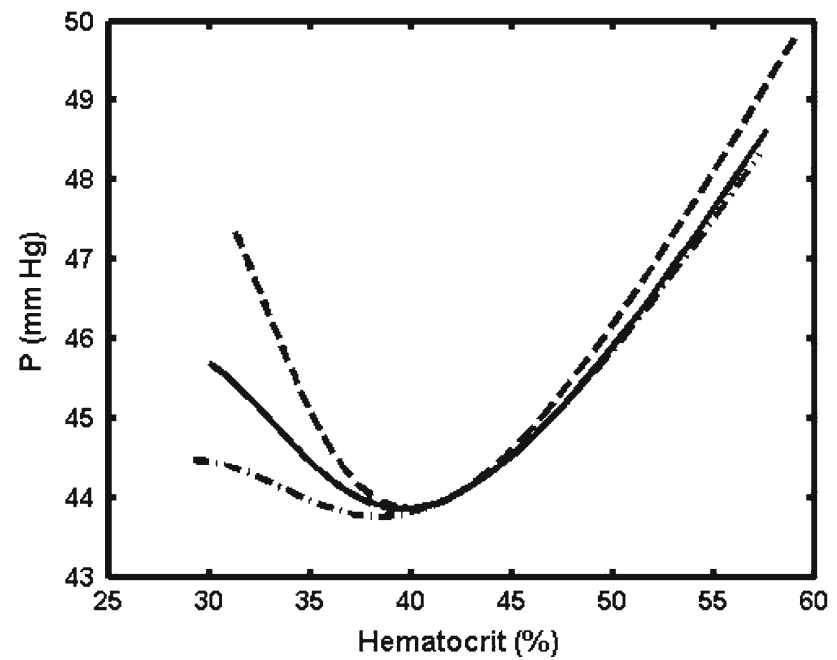

Fig. 6 Sensitivity to $C_{\text {active }}^{\prime \prime}$, the parameter that represents the range of radii where the active component of tension can act (similar to a variance on a normal distribution). The solid line, corresponding to $C_{\text {active }}^{\prime \prime}=0.354$, is the base state in Fig. 1. The dash-dot and dashed lines correspond to $C_{\text {active }}^{\prime \prime}=0.4$ and $C_{\text {active }}^{\prime \prime}=0.3$, respectively

flow in vessels account for their elasticity by reference to vascular compliance (Keener and Sneyd 2004; Loscalzo and Schafer 2002). The proposed model describes how blood pressure changes lead to vessel diameter changes determined by vascular compliance and autoregulation.

Our analysis simplifies the problem by assuming steady flow and limiting the calculation to blood pressure in a single, straight (nontapering) tube. Our mathematical analysis shows that depending on Hct (or blood viscosity) the average blood pressure of an arteriolar vessel in the microvascular network may vary by several mmHg. Within a network this effect is 
Table 1 Values of model parameters used in Fig. 1. The symbol in the value column refers to the source of this value

\begin{tabular}{lll}
\hline Constant & Value & Units \\
\hline$C_{\text {passive }}$ & $280^{*}$ & dyn/cm \\
$C_{\text {passive }}^{\prime}$ & $11.5^{*}$ & Unitless \\
$C_{\text {active }}$ & $275^{*}$ & dyn $/ \mathrm{cm}$ \\
$C_{\text {active }}^{\prime}$ & $0.750^{*}$ & Unitless \\
$C_{\text {active }}^{\prime \prime}$ & $0.354^{*}$ & Unitless \\
$C_{\text {myog }}$ & $0.0359^{*}$ & $\mathrm{~cm} /$ dyn \\
$C_{\text {shear }}$ & $0.0258^{*}$ & $\mathrm{~cm} / \mathrm{dyn}$ \\
$C_{\text {meta }}$ & $30^{*}$ & $1 /(\mu \mathrm{M} \mathrm{cm})$ \\
$C_{\text {tone }}$ & $10.7^{*}$ & Unitless \\
$S_{\text {CR }}$ & $0.45^{+}$ & $\mu \mathrm{M} \mathrm{cm}$ \\
$C_{\mathrm{p}}$ & 1.6 & $\mathrm{M}$ \\
$B$ & $2.15 \#$ & Unitless \\
$C$ & $0.875 \#$ & Unitless \\
$\mu_{\text {plasma }}$ & $0.0013 \S$ & $\mathrm{N} \mathrm{s} / \mathrm{m}^{2}$ \\
\hline
\end{tabular}

* $\{$ Carlson et al. $2008 \# 1759\}$; \#\{ries et al. $1992 \# 1536\} ; \S\{$ Koller et al. $1987 \# 1470\}$

+ Arciero et al. 2008

cumulative, and the total pressure change for the whole circulatory system would depend on the number of segments or branches in which the effect occurs. As an example, Koller et al. (1987) found in the cat sartorius muscle an average of 4 segments (or order of branches) between arcading arterioles with diameter $>25 \mu \mathrm{m}$ and capillaries.

There is no precise information on how many levels of arteriolar branching exist between the terminal arterioles and the small arteries with diameter $\sim 50 \mu \mathrm{m}$ explored in our model, and how many vessel orders will exhibit the elastic behavior of our model as we ascend the arterial tree. The number is likely to be on the order of 20 arteriolar/small artery branches or more. Whether all of these vessels would show similar behavior is not known. However, according to Kassab (2006) "it has been shown that all vascular trees examined (coronary, pulmonary, vascular systems of various skeletal muscles, mesentery, omentum, and conjunctiva) in various species (rat, hamster, cat, rabbit, pig, and human) obey a set of design rules or scaling and scaling laws." Thus, if some vessels present a specific behavior it may be assumed that, to a varying degree, this is a general feature for the circulation.

The constant flow assumption could be problematic since the circulation autoregulates flow in response to changes in the intrinsic oxygen-carrying capacity. We used this assumption solely to simplify the derivation and justify it by the very definition of autoregulation.

In our model, blood viscosity changes directly as a function of Hct. Under physiological conditions, factors such as a decrease in cardiac output (Shore and Tooke 1997) or vasoconstriction (Kassab 2006) may cause flow to decrease as Hct increases, an effect that occurs via the decrease in car- diac output (Richardson and Guyton 1959) or vasoconstriction (Jefferson et al. 2002). This effect may be present in the diabetic population since MAP initially decreases as Hct increases. The subsequent increase in MAP could be due to the increase in blood viscosity which progressively increases resistance to flow and eventually MAP. However, in normal experimental animals subjected to small Hct changes, MAP decreased and cardiac output increased significantly as Hct was initially raised. This suggests that the oxygen autoregulatory control may not have a large gain for small changes in Hct (Martini et al. 2005).

While the U-shaped relationship between MAP and Hct is present in diabetic patients and appears to be a feature of a circulatory system that adapts to acute changes in blood viscosity, it is absent in the normal population. Thus, we hypothesize that populations with anomalous MAP-Hct relationships have blood vessels with abnormal elastic and autoregulatory properties. This is expected as healthy individuals have fully functioning autoregulation, the most important variable in determining peripheral resistance, where diabetics do not. Blood viscosity in the latter therefore exerts a proportionally greater influence.

The finding that small acute changes in viscosity in healthy animals anomalously affect MAP but is not evident in the healthy human population, explored cross-sectionally as a function of the individual's Hct, indicates that there are significant differences between acute and chronic effects. In this context, effects in acute conditions were shown to be mediated by endothelial NO. Our findings indicate that in the presence of potentially diminished microvascular NO responsiveness these effects may also occur due to varying mechanical properties of blood vessels. Nevertheless, it is worthwhile emphasizing that our model, which accounts only for mechanical and fluid properties, is capable of modeling the different responses of healthy and diabetic populations.

The predictive power of the proposed model rests on the ability to measure the fitting parameters of our model in real blood vessels. This is not a trivial task, especially in vivo in humans. We present a sensitivity analysis to determine the influence of parametric uncertainty and measurement errors. The results of this analysis are presented in Figs. 2, 3, 4, 5 and 6. In general the presence of the U-shape does not appear to be particularly sensitive to the parameters other than for $C_{\text {passive }}^{\prime}$ in Fig. 3. In particular, decreasing $C_{\text {passive }}^{\prime}$ leads to a much sharper increase in pressure with increasing hematocrit, almost eliminating the observable presence of the U-shape. Figure 2 reveals that changing $C_{\text {passive }}$ results in a shift of the minimum pressure. However, the overall form of the curves does not change. As $C_{\text {passive }}$ increases, the minimum pressure decreases and is associated with lower levels of hematocrit. A similar shift in minimum pressure to lower levels of hematocrit is observable in Fig. 4 for changes in $C_{\text {active }}$. The sensitivity of pressure to $C_{\text {active }}^{\prime}$ is illustrated in Fig. 5. 
At higher levels of hematocrit, the pressure seems fairly insensitive to changes in $C_{\text {active }}^{\prime}$, whereas a more significant divergence is observable at lower hematocrit where smaller values of $C_{\text {active }}^{\prime}$ predict larger pressures. Finally, Fig. 6 shows that $C_{\text {active }}^{\prime \prime}$ appears to play a very similar role as $C_{\text {active }}^{\prime}$ with little difference in predicted pressures at larger values of hematocrit and more significant differences at lower hematocrits.

In conclusion, we show that blood pressure may be in part regulated by the interaction of blood viscosity and blood vessel mechanical properties. The changes in blood viscosity are small and related to the natural variability of this parameter in the population. The normal population does not exhibit a U-shaped MAP versus Hct relationship. The presence of this relationship in the type 2 diabetic individuals suggests that blood pressure in this population is in part determined by different elastic properties of the vessel wall and the body's lesser ability to autoregulate.

An extension of these studies is to analyze more realistic models of the vasculature, although it is not clear that increased modeling complexity will provide insights beyond the conclusions presented here. Finally, our findings suggest that in diabetic patients there may be an Hct range that corresponds to a comparatively lowered central blood pressure. While there is agreement that increased Hct and blood viscosity can be detrimental, it is apparent that lowered blood viscosity is not necessarily beneficial.

Acknowledgments This work was supported by USPHS NIH grant HLBI R01-62354.

Open Access This article is distributed under the terms of the Creative Commons Attribution Noncommercial License which permits any noncommercial use, distribution, and reproduction in any medium, provided the original author(s) and source are credited.

\section{Appendix A: Alternative closure based on the pressure-shear hypothesis}

The pressure-shear hypothesis was proposed by Pries and Secomb (2000) who studied the relationship between wall shear stress and intravascular pressure in arterioles, capillaries and venules. They reported a sigmoidal relationship between shear stress and intravascular pressure that can be written as

$\tau=\frac{C_{1}}{1+C_{3} \exp \left[-C_{2}\left(P-P_{r e f}\right)\right]}$.

This relationship was shown to hold over a wide population of different vessels as a result of structural adaptation. For Poiseuille's law (1), the wall shear stress is given by

$\tau=\frac{4 \mu Q}{\pi r^{3}}$.

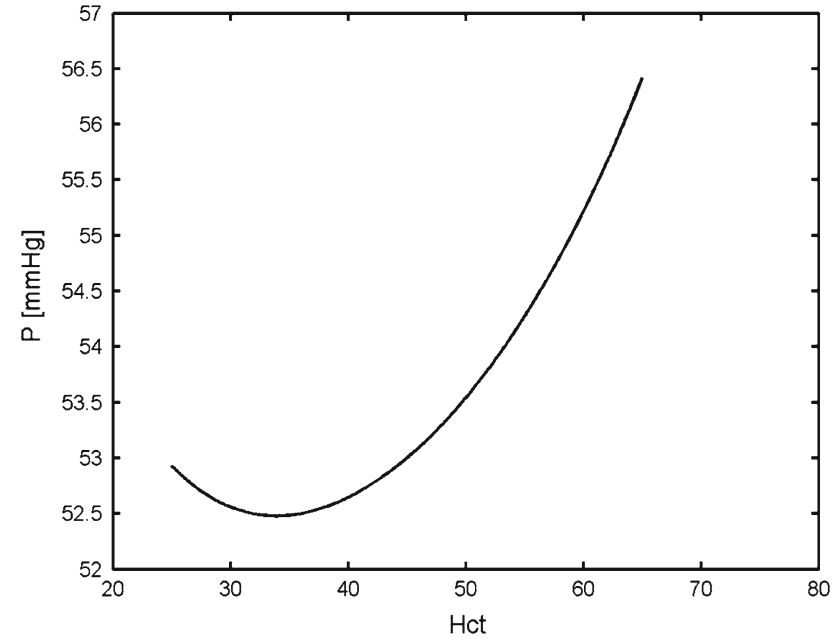

Fig. A1 Relationship between pressure (MAP) and Hct using the pressure-shear hypothesis, where the relationship between shear stress and intravascular pressure is sigmoidal. The model parameters are set to $C_{1}=100, C_{2}=2, C_{3}=1$, and $P_{\text {ref }}=20$

Combining (A1) and (A2), using (2, 3, 4, 5, 6, 7) and (10), and noting that $r$ is a function of $P$ yields a relationship between pressure and hematocrit. A sample figure, displaying the Ushape obtained with this closure approximation is presented in Fig. A1.

\section{References}

Arciero JC, Carlson BE, Secomb TW (2008) Theoretical model of metabolic blood flow regulation: roles of ATP release by red blood cells and conducted responses. Am J Physiol Heart Circ Physiol 295(4):H1562-H1571

Bertinieri G, Parati G, Ullian L (1998) Hemodilution reduces clinic and ambulatory blood pressure in polycythemic patients. Hypertension 31:848-853

Cameron JD, Cruickshank JK (2007) Glucose, insulin, diabetes and mechanisms of arterial dysfunction. Clin Exp Pharmacol Physiol 34:677-682

Carlson BE, Arciero JC, Secomb TW (2008) Theoretical model of blood flow autoregulation: roles of myogenic, shear-dependent, and metabolic responses. Am J Physiol Heart Circ Physiol 295:H1572-H1579

Carlson BE, Secomb TW (2005) A theoretical model for the myogenic response based on the mechanics of vascular smooth muscle. Microcirculation 12:327-338

Jefferson JA, Escudero E, Alfaro RT, Schoene RB (2002) Excessive erythrocytosis, chronic mountain sickness, and serum cobalt levels. Lancet 359:407-408

Kassab GS (2006) Scaling laws of vascular trees: of form and function. Am J Physiol Heart Circ Physiol 290:H894-903

Keener J, Sneyd J (2004) Mathematical physiology. Springer, New York

Koller A, Dawant B, Liu A, Popel AS, Johnson PC (1987) Quantitative analysis of arteriolar network architecture in cat sartorius muscle. Am J Physiol 253:H154-H164

Levick JR (2003) An introduction to cardiovascular physiology, 4th edn. Hodder Arnold, UK 227 
Loscalzo J, Schafer AI (2002) Thrombosis and hemorrhage. Lippincott Williams \& Wilkins, Boston MA

Malik RA, Schofield IJ, Izzard A, Austin C, Bermann G, Heagarty AM (2005) Effects of angiotensin type-1 receptor antagonism on small artery function in patients with type 2 diabetes mellitus. Hypertension 45

Martini J, Carpentier B, Chavez Negrete A, Frangos JA, Intaglietta M (2005) Paradoxical hypotension following increased hematocrit and blood viscosity. Am J Physiol Heart Circ Physiol 289:H2136$\mathrm{H} 2143$

Natali A, Toschi E, Baldeweg S, Casolaro A, Baldi S, Sironi AM, Yudkin JS, Ferrannini E (2005) Haematocrit, type 2 diabetes, and endothelium-dependent vasodilatation of resistance vessels. Eur Heart J 26:464-471

Pries AR, Neuhaus D, Gaehtgens P (1992) Blood viscosity in tube flow: dependence on diameter and hematocrit. Am J Physiol 263:H1770-H1778

Pries AR, Secomb TW (2000) Microcirculatory network structures and models. Ann Biomed Eng 28:916-921
Quarteroni A, Tuveri M, Venezianio A (2000) Computational vascular fluid dynamics: problems, models and methods. Comput Visual Sci 2:163-197

Richardson TQ, Guyton AC (1959) Effects of polycythemia and anemia on cardiac output and other circulatory factors. Am J Physiol 197:1167-1170

Salazar-Vázquez BY, Intaglietta M, Rodriguez-Morán M, GuerreroRomero F (2006) Blood pressure and hematocrit in diabetes and the role of endothelial responses in the variability of blood viscosity. Diabetes Care 29:1523-1528

Secomb TW (2008) Theoretical models for regulation of blood flow. Microcirculation 15:765-775

Shore AC, Tooke JE (1997) Microvascular function and haemodynamic disturbances in diabetes mellitus and its complications. Textbook of diabetes 1

Toy P, Feiner J, Viele MK, Watson J, Yeap H, Weiskopf RB (2000) Fatigue during acute isovolemic anemia in healthy, resting humans. Transfusion 40:457-460 